\title{
USER EXPERIENCES WITH TRADITIONAL AND 3D-PRINTED UPPER EXTREMITY PROSTHESES, DEVELOPMENT OF A COMPREHENSIVE SURVEY INSTRUMENT
}

\author{
Jennifer Mankoff $^{1}$, Saiph Savage ${ }^{2}$, Sydney Eckert ${ }^{* 3}$, Chelsea Ngo $^{* 4}$, Goeran Fiedler ${ }^{5}$ \\ ${ }^{1}$ Allen School of Computer Science \& Engineering, University of Washington, Seattle, WA, USA. \\ ${ }^{2}$ Human Computer Interaction Lab, Computer Science and Electrical Engineering Department, West Virginia University, \\ Morgantown, WV, USA. \\ ${ }^{3}$ Department of Clinical Psychology, Seattle Pacific University, WA, USA. \\ ${ }^{4}$ Western University of Health Sciences, Pediatric Therapy Network, Pomona, USA. \\ ${ }^{5}$ Department of Rehabilitation Science and Technology, University of Pittsburgh, Pittsburgh, PA, USA. \\ *33 Email: eckerts@spu.edu \\ *4 Email: csngo@westernu.edu
}

\section{INTRODUCTION}

Additive Manufacturing (AM), colloquial known as 3Dprinting, has been deemed capable to revolutionize a great number of industries, including the Health Care industry. ${ }^{1}$ In the field of upper limb prosthetics, it has been attempted to leverage the potential advantages of AM, such as crowd based design optimization, infrastructure independent fabrication, and economical material use, in the interest of providing low-cost, readily available devices to recipients whose needs were only insufficiently met by traditional approaches of device prescription and fitting. While the popular media has been quick to emphasize the potential - perceived or real - of 3D printed prostheses, clinicians have generally been less euphoric and the base of scientific evidence on questions related to these applications has been small. ${ }^{2}$

As with most research endeavors in prosthetics and orthotics, recruiting sufficient sample sizes to allow solid conclusions is a perennial challenge also in this sub-field. As a consequence, the effectiveness of the many 3Dprinted upper limb devices made by volunteers of the Enable community (Fig.1) is yet to be determined. Selfreported outcome assessment tools can somewhat mitigate the issue of low sample sizes. However, none have been applied to a wider range of device classes, to allow comparative analyses across those. We describe the development and preliminary testing of an online based survey tool to generate comparison outcome data for a wide variety of upper limb prosthetics devices, including varieties that are 3D-printed by hobbyists.

\section{METHODS}

A focus group consisting of experts from different disciplines related to upper extremity prosthesis device production was assembled to develop a comprehensive outcome survey tool, following four stages:

1.Definition of goals and target populations that will be addressed with the survey.

2.Identification of existing survey tools that can be adapted for the purposes of the eventual instrument.

3. Combination of core areas identified under 1.) and 2.) into a comprehensive questionnaire, utilizing skip logic and other methods to minimize survey length and user burden.

4.Iterative testing and refining of the draft survey to assure technical function as well as appropriateness of evaluation and reporting approaches. The group, members of which are located throughout the continental US, conducted bi-weekly online meetings over the course of several months to accomplish these objectives. The eventual survey was realized in Qualtrics Research Core (Seattle, WA) and is now available online. Data collection is currently ongoing.

\section{RESULTS}

The current survey has 107 and 108 questions respectively in the version for caregivers (capturing experiences of underage users) and for end users. Those questions are organized in 10 blocks (Table 1), some of which are only presented if previous answers suggest that they are applicable in the individual case. Completion time for the complete survey is estimated at $15-20$ minutes. 
Table 1: Overview of survey contents

\begin{tabular}{lll}
\hline Block & Description & Num. of \\
& & Questions \\
\hline 1 & Eligibility & 6 \\
2 & Demographics & 12 \\
3 & Type of device & 20 \\
$4-7$ & Usefulness of specific & 14 each \\
& device(s) & \\
8 & Problems with device & 9 \\
9 & e-Nable community & 3 \\
10 & Conclusion & 2
\end{tabular}

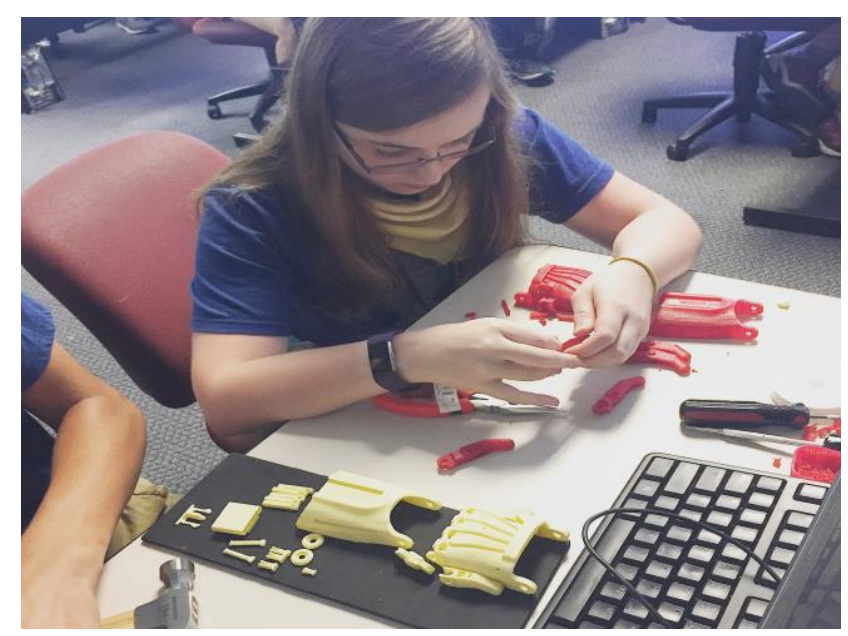

Figure 1: Volunteer assembling a 3D-printed prosthetics device.

\section{DISCUSSION}

Dependable outcome data is important to evaluate and compare novel approaches to prosthetic device provision. Survey tools can reach a large target population and have a comparably low burden of participation, which mitigates the common issue of recruiting a desirably large sample population. However, establishing the internal and external validity of such tools can be challenging. Other challenges include assuring the sensitivity and specificity of an instrument that is intended to address the same construct in a wide and diverse population or range of device classes, respectively. We have attempted to solve those challenges by developing the survey based on existing tools and leveraging the combined insights from a diverse expert panel. It is our hope that it will succeed to gather a substantial quantity of outcome data with the here presented comprehensive survey tool. This will help objectively assess the comparative effectiveness of different prosthesis concepts, including 3D-printed devices, in specific user scenarios.

\section{CONCLUSION}

A recently developed comprehensive outcome data survey for users of upper limb prosthetic devices addresses limitations of existing tools.

\section{CLINICAL APPLICATIONS}

The survey may be used for individual outcome assessment purposes over time. Aggregated data may inform prescription of conventional and novel prosthetic devices in the future.

\section{REFERENCES}

1.Giannatsis and Dedoussis. Additive fabrication technologies applied to medicine and health care: a review. Int J Adv Manuf Tech 40.1-2 (2009): 116-127. DOI: 10.1007/s00170-0071308-1

2. Jelle ten Kate, Smit, 3D-printed upper limb prostheses: a review. Breedveld, Disabil Rehabil Assist Technol. 12.3 (2017): 300-314. DOI:10.1080/17483107.2016.1253117

3. Zuniga, Jorge, et al. Cyborg beast: a low-cost 3d-printed prosthetic hand for children with upper-limb differences. BMC Res notes (2015): 8:10. DOI: 10.1186/s13104- 015-0971-9 